\title{
Synergistic Antimicrobial Effect of Chitosan Polymers and Oligomers
}

\author{
Maha Attjioui, ${ }^{1}$ Dominique Gillet, ${ }^{2}$ Nour Eddine El Gueddari, ${ }^{1}$ and Bruno M. Moerschbacher ${ }^{1, \dagger}$ \\ ${ }^{1}$ Institute for Biology and Biotechnology of Plants, University of Münster, Münster, Germany \\ ${ }^{2}$ Gillet Chitosan SAS, La Ville Es Comte, 22350 Plumaudan, France
}

Accepted 8 March 2021.

\begin{abstract}
This study evaluated the efficacy of the combined application of well-characterized chitosan polymer (degree of acetylation $=$ $10 \%$, degree of polymerization $[\mathrm{DPn}]=90$, and dispersity $\left[\bigoplus_{\mathrm{DP}}\right]=2.8$ ) and oligomers (partially acetylated chitosan polymers and oligosaccharides [paCOS] $)(\mathrm{DP}=2$ to 17) on conidia germination and mycelial growth of Fusarium graminearum, the major causal agent of Fusarium head blight in wheat. The polymer alone showed a higher inhibitory effect than the paCOS mixture alone, with half-maximal inhibitory concentrations of less than $50 \mu \mathrm{g} \mathrm{ml}^{-1}$ and more than $100 \mu \mathrm{g}$ $\mathrm{ml}^{-1}$, respectively. Using time-lapse microscopy, we also showed that paCOS did not affect conidia germination at $50 \mu \mathrm{g} \mathrm{ml}^{-1}$, whereas chitosan polymer at the same concentration led to a delay in germination and in elongation of germ tubes. Scanning electron microscopy was used to observe the chitosan-induced changes in hyphal morphology. Surprisingly, the combination of chitosan polymer and paCOS led to strong synergistic effects in inhibiting conidia germination and fungal growth, as quantified by both the Abbot and Wadley equations. To our knowledge, this is the first report on a synergistic effect of a combination of chitosan polymers and oligomers, also highlighting for the first time the importance of $\bigoplus_{\mathrm{DP}}$ when studying structure-function relationships of functional biopolymers such as chitosan. The consequences of this finding for the improvement of chitosan-based antimicrobial or plant protective products are discussed. Given the economic importance of $F$. graminearum, this study suggests that the combination of chitosan polymer and oligomers can be used to support an efficient, sustainable plant protection strategy.
\end{abstract}

Keywords: chitosan, Fusarium graminearum, oligosaccharides, polymer, synergism

${ }^{\dagger}$ Corresponding author: B. M. Moerschbacher; moersch@uni-muenster.de

Nour Eddine El Gueddari is deceased.

Funding: Support was provided by the German Academic Research Council (Deutscher Akademischer Austauschdienst) through a doctoral scholarship to M. Attjioui.

*The $e$-Xtra logo stands for "electronic extra" and indicates that supplementary materials and supplementary videos are published online.

The author(s) declare no conflict of interest.

(i) Copyright $\odot 2021$ The Author(s). This is an open access article distributed under the CC BY 4.0 International license.
Fusarium graminearum is primarily a pathogen of cereal plants, particularly wheat, causing crown and root rot, as well as Fusarium head blight (FHB). FHB has emerged as an economically important plant disease during the last decades, due to reduced grain yields and quality as well as the production of mycotoxins which are harmful to humans and animals. Moreover, FHB is a challenging disease to control, on the one hand because of the lack of highly resistant or tolerant wheat cultivars, and on the other hand because of the problems faced with conventional treatments such as their toxicity, side effects, resistance development, and high costs. Therefore, alternative approaches to prevent FHB need to be developed. One new approach is the use of functional biopolymers from renewable resources as biopesticides (Niekraszewicz et al. 2012) such as chitosans, the partially deacetylated counterparts of one of the most abundant renewable resources on earth, chitin (van den Broek and Boeriu 2020).

The term chitosan or, rather, chitosans, refers to a heterogeneous group of copolymers consisting of $\mathrm{N}$-acetyl-D-glucosamine (GlcNAc) and D-glucosamine $(\mathrm{GlcN})$ units, linked together by $\beta-(1 \rightarrow 4)$ glycosidic bonds, differing in their degrees of polymerization (DP) and acetylation (DA) (Cord-Landwehr et al. 2020a; Raafat and Sahl 2009; Younes and Rinaudo 2015). Because of their multiple positive charges at slightly acidic $\mathrm{pH}$ values due to the GlcN units, combined with their partially hydrophobic character due to the GlcNAc units, chitosans possess unique physiological and biological properties (Cord-Landwehr et al. 2020b; Wattjes et al. 2020). In addition, their inherent property of being environmentally friendly if the production process is well managed, biocompatible in many contexts, and biodegradable in the environment make them attractive for many commercial applications (Halim et al. 2012; Muñoz et al. 2018).

Chitosans have been explored for many agricultural uses; for example, as inducers of plant defense responses (Conrath et al. 1989; Hadwiger and Beckman 1980; Köhle et al. 1985; Vander et al. 1998); as plant growth promoters (El Hadrami et al. 2010); and as antimicrobial agents against a large number of plant pathogens, including bacteria (Shigemasa and Minami 1996; Sudarshan et al. 1992; Tsai and Su 1999), viruses (Iriti and Varoni 2015), and fungi (Allan and Hadwiger 1979; Benhamou 1992; Rabea et al. 2003). Some studies investigated the effect of chitosan for the control of the fungal pathogen $F$. graminearum. For instance, Reddy et al. (1999) showed that seed treatment of spring wheat with chitosan induced resistance to $F$. graminearum and improved seed quality, confirmed later by studies on durum wheat, where seed treatment with chitosan elicited an induction of enzymes involved in defense mechanisms and a decrease in disease severity (Orzali et al. 2014). However, studies on a possible 
direct antimicrobial effect of chitosan on $F$. graminearum, which might provide insight into fundamental mechanisms of its plant protective effects, are limited. For example, Brunel et al. (2013) showed that the combination of copper and chitosan in the form of nanoparticles led to stronger growth inhibition of $F$. graminearum when compared with copper or chitosan alone in vitro, and Kheiri et al. (2016) showed the antifungal effect of chitosan and chitosan nanoparticles in vitro and in vivo. However, most of these studies have been conducted with only a single and often poorly defined chitosan and, thus, have not addressed the issue of structurefunction relationships of chitosans, which is important to better understand their mode of action and to optimize their application (Basa et al. 2020; Cord-Landwehr et al. 2020b; Hamer et al. 2014; Vander et al. 1998; Wattjes et al. 2020).

To help elucidate these structure-function relationships, we investigated the efficacy of well-characterized, partially acetylated chitosan polymers and oligosaccharides (paCOS) alone and in combination in vitro for their antifungal effect against the economically important seedborne pathogen $F$. graminearum, the causal agent of FHB in cereals.

\section{RESULTS}

\section{Characterization of the chemical hydrolysate fractions.}

The basis of our study was a commercially available chitosan preparation (produced by Mahtani Chitosan Pvt. Ltd. India, under guidance of Dr. Dominique Gillet) which was developed in a decade-long public-private collaboration aiming to optimize the performance of chitosan-based agrobiologics. Based on academic results concerning structure-function relationships of defined chitosans produced at lab scale (Cord-Landwehr et al. 2020b; Moerschbacher 2005; Wattjes et al. 2020), an economically viable process has been developed that yields a chitosan preparation which has proven extremely successful as a dualeffect biostimulant and biopesticide in different crops, promoting plant growth and development as well as increasing disease resistance and stress tolerance. This chitosan preparation was obtained by partial chemical depolymerization of a microcrystalline chitosan polymer which we characterized as having a medium DP (average DPn = approximately 300), a low dispersity $\left(\bigoplus_{\mathrm{DP}}=1.24\right)$, and a low DA (average DA $=10 \%$ ), using state-of-the-art analytical techniques (Supplementary Fig. S1). Size exclusion chromatography (SEC) of the hydrolysate (average $\mathrm{DPn}=70$, average $\mathrm{DA}=10 \%$ ) revealed that it consists of a

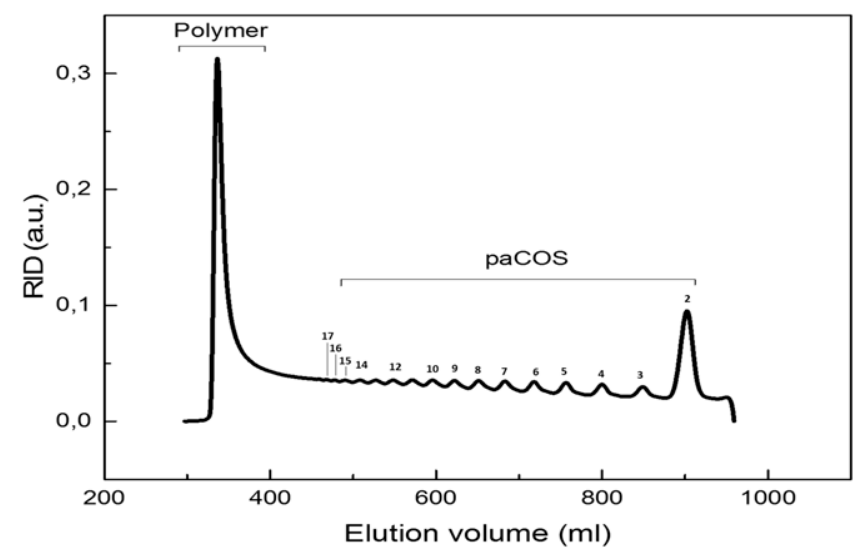

Fig. 1. Size exclusion chromatography analysis of the chemical hydrolysate obtained from the hydrolysis of the parent chitosan (degree of acetylation $=10 \%$, degrees of polymerization $=300$, and dispersity $=1.24$ ) The hydrolysate is composed of both chitosan polymer and chitosan oligosaccharides (partially acetylated chitosan oligosaccharides [paCOS]). polymeric fraction $(43 \%, \mathrm{wt} / \mathrm{vol})$ and a mixture of chitosan oligomers (paCOS) $(55 \%, \mathrm{wt} / \mathrm{vol})$ (Fig. 1). Analysis of the polymeric fraction using high-performance SEC coupled with refractive index detector and multi-angle laser light scattering (HP-SEC-RID-MALLS) showed that it has a low molecular weight (average DPn $=90)$, a higher dispersity $\left(\bigoplus_{\mathrm{DP}}=2.85\right)$, and a low DA (average DA $=10 \%$ ) (Supplementary Fig. S1). Analysis of the paCOS fraction using matrix-assisted laser desorption-ionization time of flight mass spectrometry (MALDITOF-MS) showed that the paCOS mixture has a DP range between 2 and 17. Because both the total hydrolysate and its polymeric fraction have a DA of $10 \%$, so does the oligomeric fraction.

\section{Antimicrobial activity as a function of DP.}

In order to investigate the influence of the chitosan's DP on the antimicrobial effect, we quantified the growth of fungal mycelium in the presence of increasing amounts of the different chitosan samples in a microtiter plate-based bioassay (Fig. 2). The minimum inhibitory concentration (MIC, defined as the lowest concentration that fully inhibits fungal growth) and the half-maximal inhibitory concentration $\left(\mathrm{IC}_{50}\right.$, defined as the concentration that is required for $50 \%$ inhibition of fungal growth) of a chitosan were assessed after 5 days of incubation (Supplementary Table S1). When we compared the antimicrobial activity of the high-DP parent polymer and its hydrolysate, both showed similar inhibitory activities, with MIC values of approximately $100 \mu \mathrm{g} \mathrm{ml}^{-1}$ and $\mathrm{IC}_{50}$ values of approximately $50 \mu \mathrm{g}$ $\mathrm{ml}^{-1}$. The low-DP polymeric fraction was slightly more active than the parent polymer and the total hydrolysate, while the chitosan oligomers were clearly less active, with a MIC of more than $200 \mu \mathrm{g} \mathrm{ml}^{-1}$ and an $\mathrm{IC}_{50}$ of more than $100 \mu \mathrm{g} \mathrm{ml}{ }^{-1}$. Because the total hydrolysate consists of $43 \%$ (wt/vol) polymer and $55 \%$ (wt/vol) paCOS, one should expect that their mixture, the total hydrolysate, would be less active than the polymeric fraction alone. However, this was clearly not the case.

As an example, the hydrolysate at $100 \mu \mathrm{g} \mathrm{ml}^{-1}$ contains polymer at $43 \mu \mathrm{g} \mathrm{ml}{ }^{-1}$. This amount of polymeric fraction alone leads to approximately $50 \%$ inhibition, while the paCOS at 55 $\mu \mathrm{g} \mathrm{ml}{ }^{-1}$ present in the hydrolysate would be expected to be inactive. Thus, we would expect a 50\% growth inhibition for the hydrolysate at $100 \mu \mathrm{g} \mathrm{ml}^{-1}$ but, in fact, we observed a full inhibition of fungal growth. Similarly, hydrolysate at $50 \mu \mathrm{g}$ $\mathrm{ml}^{-1}$ would be expected to be inactive because both fractions are inactive at their respective concentrations (polymer at $22 \mu \mathrm{g}$ $\mathrm{ml}^{-1}$ and paCOS at $28 \mu \mathrm{g} \mathrm{ml} \mathrm{m}^{-1}$ ) but, in fact, we observed a $50 \%$ inhibition, strongly hinting at a synergistic rather than an additive effect. Before analyzing this unexpectedly high antimicrobial activity of the hydrolysate mixture further in a more quantitative way, we examined the morphology of the fungus in the presence of the different chitosan samples.

\section{Time-lapse imaging of conidia germination.}

We followed conidia germination during $24 \mathrm{~h}$ in the presence of the mixture or its two components, using time-lapse microscopy (Fig. 3). The results show that, at a concentration of $50 \mu \mathrm{g}$ $\mathrm{ml}^{-1}$, chitosan oligosaccharides did not affect conidia germination, because conidia germinated with the same speed as the control (Supplementary Videos S1 and S2). However, conidia treated with chitosan polymer at the same concentration showed a delay in the germination and in the elongation of the germ tube (Supplementary Video S3). In the presence of both polymer and paCOS (both at $50 \mu \mathrm{g} \mathrm{ml}{ }^{-1}$ ) (i.e., mimicking the total hydrolysate at $100 \mu \mathrm{g} \mathrm{ml}^{-1}$ ), most of the conidia did not germinate but visibly changed color (Supplementary Video S4). Abnormal germination, deformation of the germ tube, and multiple polar initiation points were also observed in some conidia 
treated with the mixture of chitosan oligomers and polymers (Supplementary Fig. S2).

\section{Effect of chitosans on the ultrastructure \\ of $F$. graminearum}

Similarly, scanning electron microscopy (SEM) examination (Fig. 4) showed that nontreated hyphae of $F$. graminearum had regular, intact shapes with smooth surfaces, while considerable morphological changes were observed in all chitosan-treated hyphae, including hyphal deformation and shrinkage in the presence of polymer and hyphal surface flaking in paCOS-treated samples. Both effects were also observed when the two treatments were combined.

\section{Quantitative analysis of the synergistic effect.}

Synergism can be quantified using different algorithms, and the selection of the most appropriate one depends on the mode of action of the two compounds (Levy et al. 1986). If both compounds have different modes of action, the Abbott equation can be used to calculate a synergism factor (Abbott 1925). On the other hand, if the two compounds have the same mode of action, the synergism factor can be calculated using the method of Wadley (1945). However, as discussed below, we cannot safely predict whether chitosan polymers and oligomers have different or identical modes of action. Therefore, we calculated the synergism factor using both algorithms. The Wadley equation required us to collect more data on the antimicrobial effect of different mixtures of the two components. We designed the experiments in such a way that we added a fixed amount of paCOS $\left(50 \mu \mathrm{g} \mathrm{ml}^{-1}\right)$ to different amounts of chitosan polymer ( 0 to $110 \mu \mathrm{g} \mathrm{ml}^{-1}$ ) in increments of the equivalent dose of polymer $\left(20 \mu \mathrm{g} \mathrm{ml}^{-1}\right)$. The equivalent dose of polymer is that amount of polymer that gives the same antifungal effect as the added amount of oligomer $\left(50 \mu \mathrm{g} \mathrm{ml}^{-1}\right)$; in our case, the highest concentration that had no inhibitory effect by itself (Fig. 2B).

The growth inhibition of $F$. graminearum caused by the polymer alone in different concentrations and in combination with a low dose of paCOS, which in itself is not inhibitory, is shown in Figure 5. In the absence of paCOS, chitosan polymer at $30 \mu \mathrm{g} \mathrm{ml}^{-1}$ had no influence on fungal growth, and $50 \mu \mathrm{g} \mathrm{ml}^{-1}$ reduced fungal growth by approximately $20 \%$ only. However, in the presence of paCOS, chitosan polymer at $30 \mu \mathrm{g} \mathrm{ml} \mathrm{m}^{-1}$ inhibited fungal growth by approximately $70 \%$, and the polymer at even as little as $10 \mu \mathrm{g} \mathrm{ml} \mathrm{m}^{-1}$ reduced fungal growth by approximately $30 \%$, while $50 \mu \mathrm{g} \mathrm{ml}^{-1}$ reduced fungal growth by almost $90 \%$. These results clearly show a synergistic effect

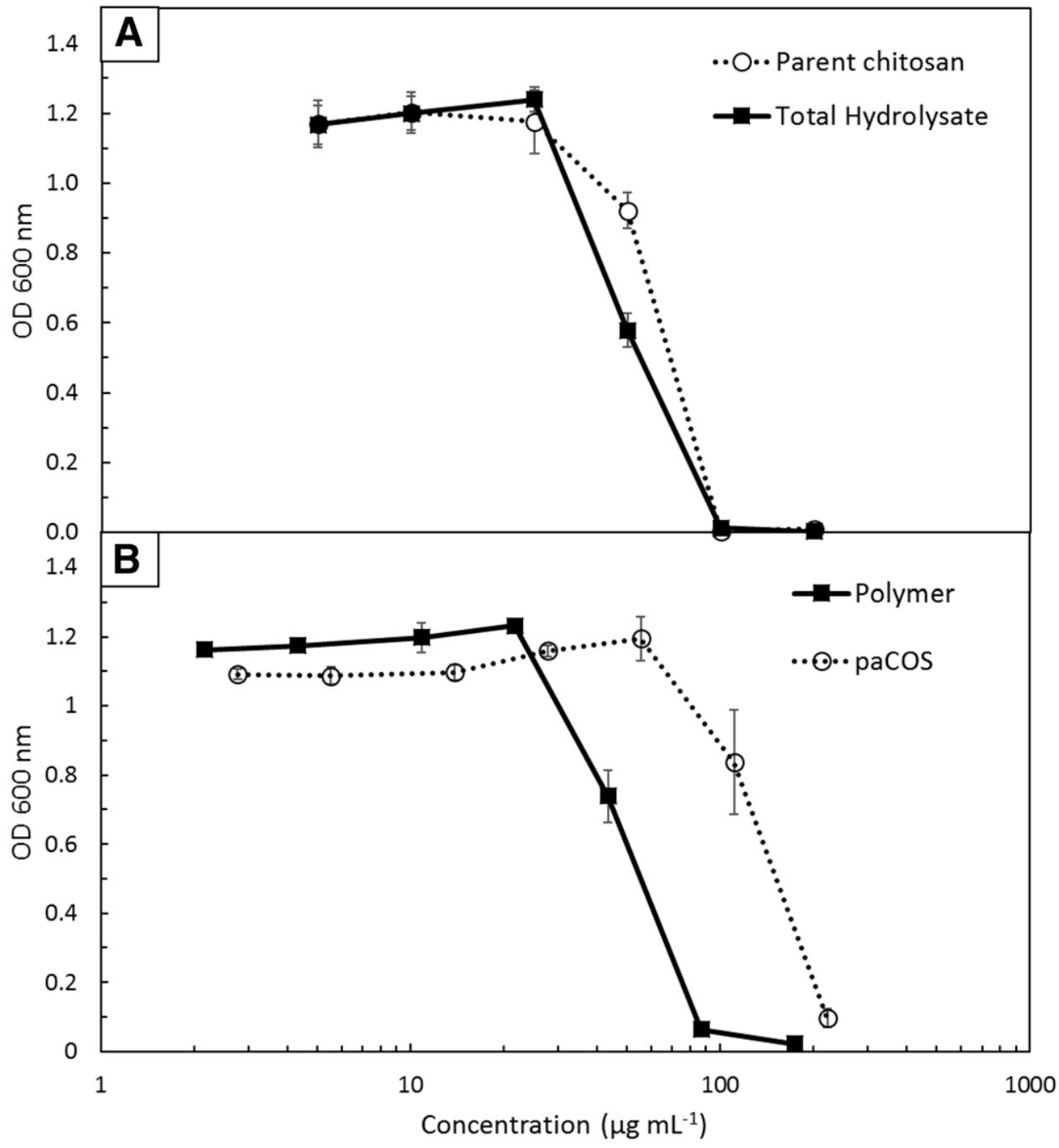

Fig. 2. Dose-response relationships for the inhibitory effect of chitosans on the growth of Fusarium graminearum after $96 \mathrm{~h}$. A, Parent chitosan polymer before hydrolysis (degree of acetylation $[\mathrm{DA}]=10 \%$, degrees of polymerization $[\mathrm{DPn}]=300$, and dispersity $\left[\mathrm{Ð}_{\mathrm{DP}}\right]=1.24$ ) and total hydrolysate mixture $(\mathrm{DA}=10 \%$ and $\mathrm{DPn}=70)$ and $\mathbf{B}$, polymeric $\left(\mathrm{DA}=10 \%, \mathrm{DPn}=90\right.$, and $\left.\mathrm{Ð}_{\mathrm{DP}}=2.8\right)$ and oligomeric fraction (partially acetylated chitosan oligosaccharides $[\mathrm{paCOS}], \mathrm{DA}=10 \%$, and $\mathrm{DP}=2$ to 17 ) of the hydrolysate. $\mathrm{OD}=$ optical density. Data shown are mean values \pm standard deviation of triplicate determinations in three independent experiments. 
in the antifungal activities of chitosan oligomers and polymers, as also quantified by synergy calculations using both the Wadley and the Abbott methods (Supplementary Table S2). In both methods, a synergy factor of 1 would indicate additivity rather than synergism, while values below 1 or above 1 indicate antagonism or synergism, respectively. Using both methods, strongly positive values indicated strong synergism between chitosan polymer and oligomers, with synergy factors reaching 15 and 78 according to Wadley and Abbott, respectively.

\section{DISCUSSION}

In our study, we showed that all chitosan treatments had direct antifungal effects against $F$. graminearum, affected conidia germination, and caused ultrastructural modifications. The antifungal activity of the oligomers was weaker than that of the polymers, and smaller polymers had slightly stronger activity than larger polymers, corroborating previous findings (Jeon et al. 2001; Moerschbacher 2005; Park et al. 2008). More importantly, chitosan polymers and oligomers showed a strong synergistic effect when combined. Fungistatic or fungicidal effects of chitosans against various species of fungi both in vitro and in situ have been reported by many authors (Allan and Hadwiger 1979; Benhamou and Theriault 1992; Laflamme et al. 1999; Rabea et al. 2003; Reddy et al. 1998; Roller and Covill 1999). Chitosan showed significant results in reducing fungal growth, associated with morphological and ultrastructural alterations of hyphae
(El Ghaouth et al. 1992) and disorganization of the cells (Benhamou 1992); chitosan also inhibited spore germination (Plascencia-Jatomea et al. 2003), entered fungal spores, and caused cell lysis (Palma-Guerrero et al. 2009). Also, it has been shown that structural properties of the chitosan used, such as the DP, are important parameters affecting its antifungal activity (Badawy et al. 2006; Hernández-Lauzardo et al. 2008; Li et al. 2011; Moerschbacher 2005; Oliveira et al. 2008). However, to our knowledge, this is the first report of a synergistic effect of chitosan polymers and oligomers. This is of considerable significance given that chitosan samples are always mixtures of molecules, often widely differing in their DP or molecular weight; however, the parameter $\mathrm{D}_{\mathrm{DP}}$ which describes this dispersity (Cord-Landwehr et al. 2020a) is very rarely mentioned in articles describing structure-function relationships of chitosans and, to our knowledge, its possible influence on the outcome of bioactivity assays has never been investigated.

Our results confirm that the efficacy of chitosan depends on its molecular weight. In our study, we analyzed the doseresponse relationship of three chitosans with the same DA $(10 \%)$ and different molecular weights - parent chitosan (DPn = $300, \bigoplus_{\mathrm{DP}}=1.24$ ), chitosan polymers with low molecular weight $\left(\mathrm{DPn}=90, \mathrm{Ð}_{\mathrm{DP}}=2.8\right)$, and paCOS $(\mathrm{DP}<20)$-against $F$. graminearum and found that small chitosan polymers were slightly more active than the large parent chitosan polymer, while paCOS were least active. The higher antifungal activity of small polymers compared with large polymers has been reported

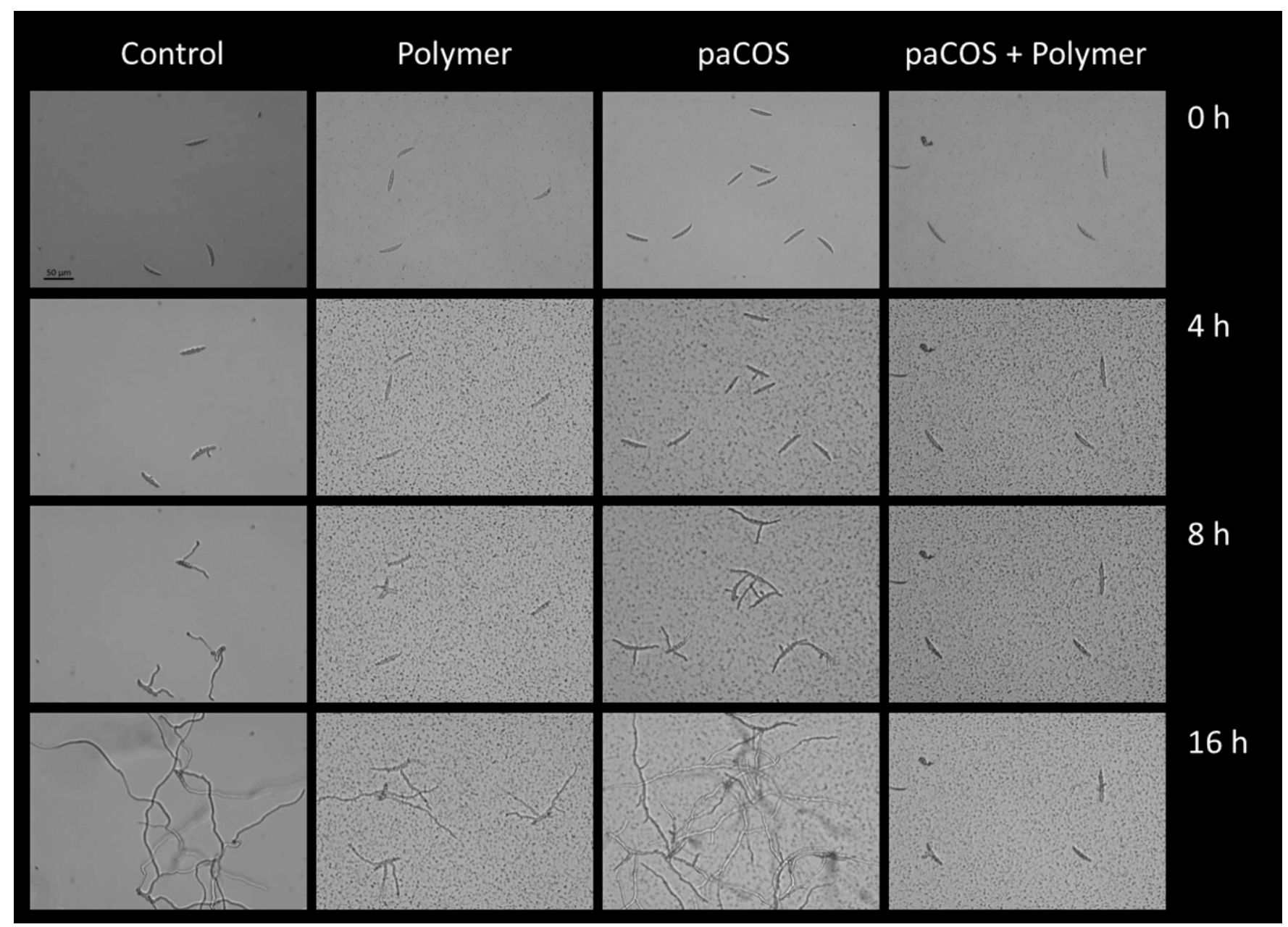

Fig. 3. Time-lapse images showing conidia germination as well as germ tube and hyphal development of nontreated Fusarium graminearum conidia (Control), conidia treated with chitosan polymer alone at $50 \mu \mathrm{g} \mathrm{ml}{ }^{-1}$ (Polymer), with only partially acetylated chitosan oligosaccharides (paCOS) at $50 \mu \mathrm{g} \mathrm{ml}{ }^{-1}$, or with the combination of paCOS and chitosan polymer, both at $50 \mu \mathrm{g} \mathrm{m}{ }^{-1}$ (paCOS + Polymer), at different time points. 
before (Alburquenque et al. 2010; Hirano and Nagao 1989; Li et al. 2008; Moerschbacher 2005; Park et al. 2008; Rhoades and Roller 2000; Wattjes et al. 2020). Most likely, this is caused by the higher mobility of the smaller polymers, particularly within the fungal cell wall. The antifungal activity of chitosans is agreed to be mainly due to their polycationic structure, because the cationic groups might interact with anionic components of the negatively charged cell wall of the fungus and destabilize the phospholipid-containing plasma membrane (Hirano and Nagao 1989; Park et al. 2008; Rabea et al. 2003; Wattjes et al. 2020). The latter effect can be expected to be much stronger for chitosan polymers which bind to a larger cell surface area, thus deforming the membrane phospholipids more strongly than chitosan oligomers (Kauss et al. 1989). In fact, Jeon et al. (2001) showed that chitosan with molecular weights higher than or approximately $10 \mathrm{kDa}$ had stronger membrane-disrupting abilities than chitosans of lower molecular weights. These results agree with our findings because the membrane cut off used for the separation of chitosan polymer and paCOS was $10 \mathrm{kDa}$.

However, other studies have observed higher antifungal activity for paCOS than for long-chain chitosan polymers, conflicting with our findings (Kim and Rajapakse 2005). Such conflicting results when assaying biological activities of chitosans are very frequent and have been attributed to the use of poorly defined chitosans (El Gueddari and Moerschbacher 2004; Nars et al. 2013; Wattjes et al. 2020; Winkler et al. 2017). Even in cases where some structural characteristics of the chitosans used are given, these are almost always restricted to the DP or molecular weight and the DA. However, these values invariably are average values, and the molecular weight distribution or dispersity of the samples used is almost never indicated. Our finding of synergistic effects of chitosans with different molecular weights adds a further level of complexity to the antifungal activities of chitosans, highlighting the importance of measuring and indicating at least the dispersity when assaying the biological activity of chitosan samples. However, it must be noted that the dispersity $\bigoplus_{\mathrm{DP}}$ can only indicate the dispersity of a chitosan polymer sample or of the polymeric fraction of a mixture of polymers and oligomers but does not inform about the presence or absence of oligomers itself, or of the dispersity of the oligomeric fraction, if present. Thus, a thorough DP analysis of any chitosan sample requires an initial check for the presence or absence of polymers and oligomers, followed by careful separate characterization of both fractions, if present.

The most important finding of the present work is the synergistic effect of the combination of chitosan polymers and paCOS. Our results show that the combination of inactive concentrations of paCOS $\left(50 \mu \mathrm{g} \mathrm{ml}^{-1}\right)$ and chitosan polymer (10 and $30 \mu \mathrm{g} \mathrm{ml}^{-1}$ ) leads to strong synergistic effects, resulting in 29 to $71 \%$ fungal inhibition, respectively. To safely distinguish between additive and synergistic effects is not trivial, particularly in a case like this where structurally similar and, thus, possibly also functionally similar compounds are combined (Levy et al. 1986). Two different methods have been proposed to calculate a synergy factor. The method of Abbott applies in case the two compounds have different modes of action (Abbott 1925), while the method of Wadley must be used if both compounds have the same mode of action (Wadley 1945). In the case of chitosan oligomers and polymers, the modes of action are unknown at present; they may act via the same or via

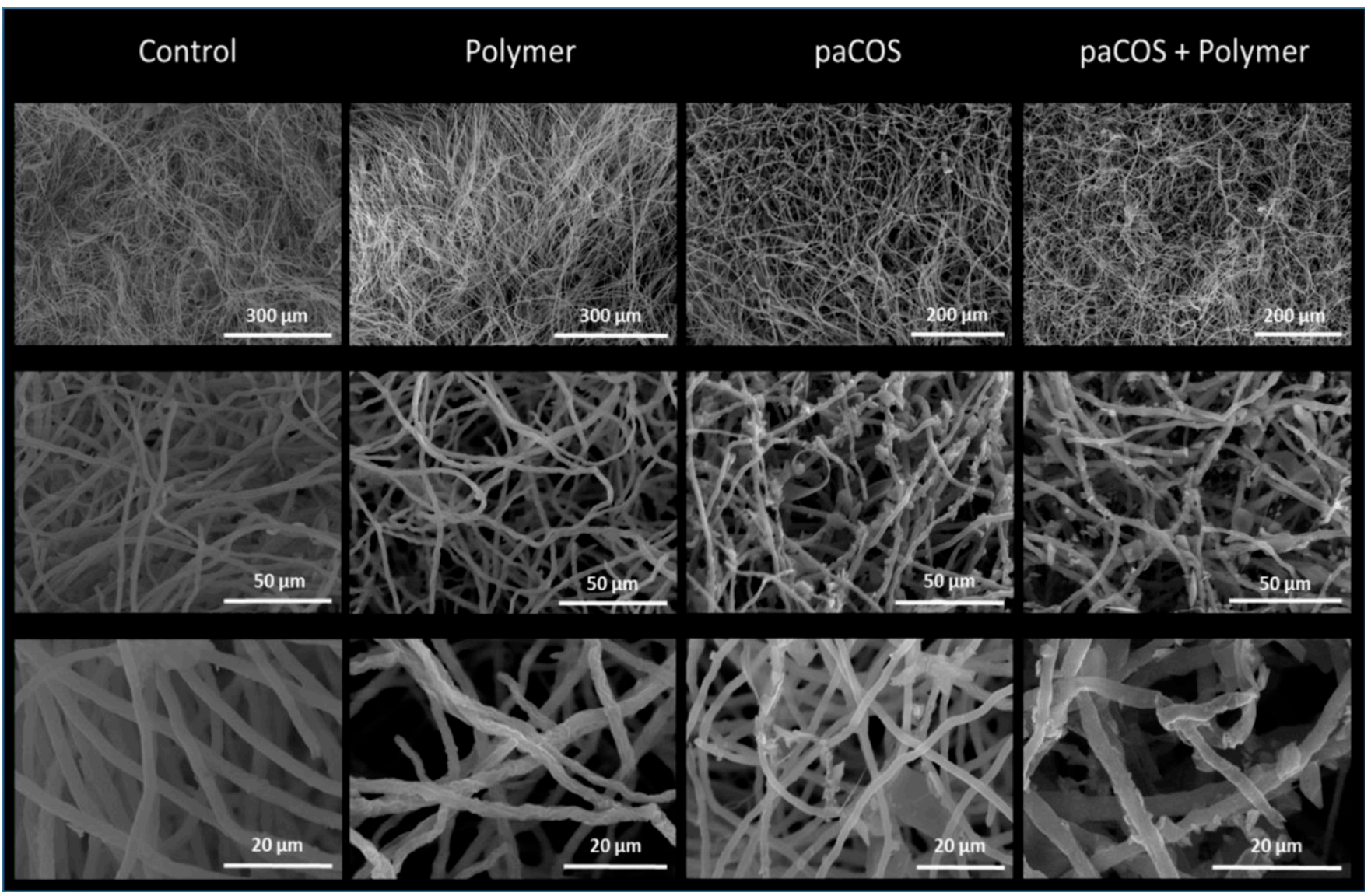

Fig. 4. Scanning electron microscopy images showing hyphae developed from nontreated Fusarium graminearum conidia (Control), hyphae grown in the presence of chitosan polymer alone at $50 \mu \mathrm{g} \mathrm{ml}^{-1}$ (Polymer), only partially acetylated chitosan oligosaccharides (paCOS) at $50 \mu \mathrm{g} \mathrm{ml}{ }^{-1}$, or the combination of paCOS and the chitosan polymer, both at $50 \mu \mathrm{g} \mathrm{ml} \mathrm{l}^{-1}$ (paCOS + Polymer), after 10 days. 
different mechanisms. One might speculate that the oligomers are taken up and act intracellularly, while the polymers are not, thus acting on the cell surface. Therefore, we performed the measurements in such a way that both methods of calculation can be applied. In both cases, the synergistic effect was confirmed. Because of the different assumptions and equations, the synergy factor differs, and it is most pronounced at different mixing ratios, as expected. A synergistic effect of chitosan with other antifungal compounds such as copper (Brunel et al. 2013), organic fungicides (Alburquenque et al. 2010; Rahman et al. 2014), and plant extracts (Putriningrum et al. 2016) has been observed; however, this is the first time that different chitosans have been shown to act in synergy with each other.

At this point, we can only speculate about the underlying reason for the observed synergistic effect (Fig. 6). In this respect, it is interesting to note that an analysis of the spent medium showed that $F$. graminearum produces chitosanolytic enzymes in the presence of paCOS and the polymer at low concentrations (Supplementary Fig. S3). One possible explanation for the synergistic effect would assume that the most strongly antifungal chitosans are very large oligomers or, depending on the definition of oligomers and polymers (Cord-Landwehr et al. 2020b; Naqvi and Moerschbacher 2017; Wattjes et al. 2020), very small polymers, consistent with the observation that the antifungal activities of chitosans first increase, then decrease with increasing molecular weight (Moerschbacher 2005). The chitosanolytic enzymes might rather quickly degrade and, thus, inactivate chitosan oligomers, whereas they might slowly degrade and, thus, activate chitosan polymers. The combination of an immediate but transient antifungal effect of oligomers with a sustained release of the most highly active large chitosan oligomers from the polymers might be responsible for the observed synergistic effect (Fig. 6A). In this case, both fractions would have the same mode of action, and the Wadley equation would be adequate for calculating the synergy factor.

A second hypothesis could be that the polymer and paCOS have different modes of action, in which case the Abbot

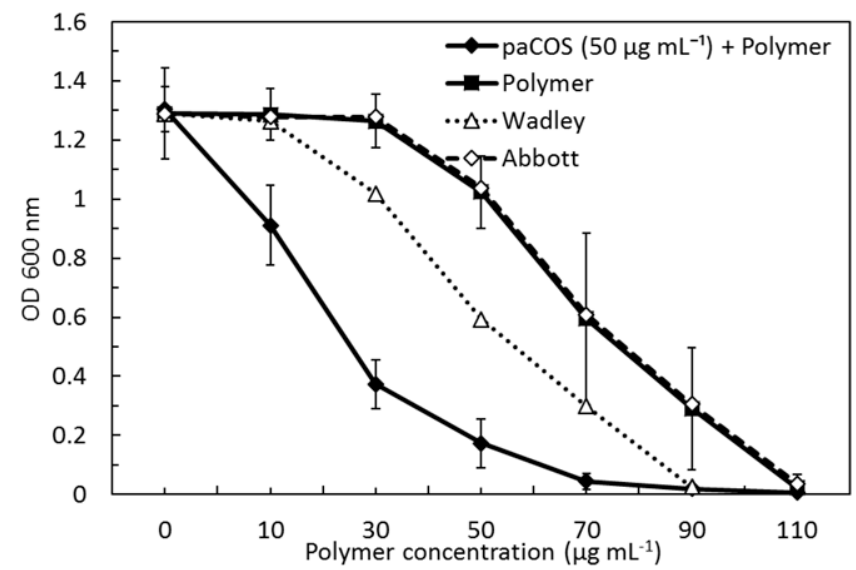

Fig. 5. Dose-response curves of the influence of chitosan polymer alone (Polymer) and in combination with a fixed dose of chitosan oligosaccharides (partially acetylated chitosan oligosaccharides [paCOS]) (50 $\mu \mathrm{g}$ $\mathrm{ml}^{-1}$ ) on the growth of Fusarium graminearum after $96 \mathrm{~h}$ (i.e., in the mixture, when Polymer $=0 \mu \mathrm{g} \mathrm{ml}^{-1}$ and $\mathrm{paCOS}=50 \mu \mathrm{g} \mathrm{ml} \mathrm{m}^{-1}$ ) and the hypothetical dose-response curves for the expected effects of the combination of chitosan Polymer and paCOS if there were no synergy between them, assuming that both components have different modes of action (Abbott equation) or the same mode of action (Wadley equation). $\mathrm{OD}=$ optical density. Clearly, the experimentally observed growth inhibition caused by the mixtures is much stronger than the expected one, regardless of the algorithm used, indicating strong synergism. Data shown are mean values \pm standard deviation of triplicate determinations in three independent experiments. equation would have to be used for synergy factor calculation. Chitosan polymer, because of its long chain, can interact with the membrane, causing membrane permeabilization, and this may allow paCOS to enter into the cell and interact with intracellular components (Fig. 6B). Further experiments are required to verify or refute either hypothesis. Clearly, the possible enzymatic processing of the chitosans applied by secreted and, possibly, chitosan-induced chitosanolytic enzymes deserves further attention. In both cases, however, a clear synergistic effect of the two fractions is observed, with obvious consequences for the design of chitosan-based plant protectants or preservatives (e.g., in food, feed, or cosmetics). Particularly when applied as a foliar spray in plant protection, the use of large polymers is potentially problematic because it can easily lead to aggregation and nozzle blockage. Using an equally bioactive mixture of small polymers and oligomers instead can avoid this problem.

We would like to mention that the chitosan used in this study is a chitosan preparation produced commercially at industrial scale. Its composition was based on extensive investigations of molecular structure-function relationships performed using welldefined chitosan polymers and oligomers produced at lab scale (Moerschbacher 2005). Using such chitosans on an industrial level for field applications would be prohibitively expensive. Therefore, once we knew which chitosans are best for plant strengthening and which ones have the highest antimicrobial activities, we designed an economically viable process to produce a chitosan preparation reliably containing both types of bioactive chitosans. The finding described here of a synergistic effect of chitosan oligomers and polymers was unexpected but certainly adds to the efficacy of the product. Our study, thus, also shows that the translation between academic research and commercial product development is a mutual process from which both sides can profit.

\section{Conclusion.}

In conclusion, we showed that the efficacy of chitosan against $F$. graminearum depends on the molecular weight, where small polymers had a better antifungal activity than large polymers or oligomers. Most importantly, the combination of chitosan polymer and oligomers showed a strong synergistic effect in inhibiting conidia germination and fungal growth, and in causing morphological changes in fungal ultrastructure. This finding highlights the thus-far-neglected importance of the molecular weight dispersity of a chitosan solution as a crucial factor influencing its bioactivity. This new insight has significant bearings on the development and improvement of chitosan-based treatments in plant disease protection as well as chitosan-based preservatives for food, feed, or cosmetics.

\section{MATERIALS AND METHODS}

\section{Chitosan characterization.}

Parent chitosan $\left(\mathrm{DA}=10 \%, \mathrm{DPn}=300, \mathrm{D}_{\mathrm{DP}}=1.24\right)$ from shrimp shell wastes and its chemically digested, water-soluble hydrolysate $(\mathrm{DA}=10 \%, \mathrm{DPn}=70)$ were provided by Mahtani Chitosan Pvt. Ltd. (Gujarat, India). DP and $\bigoplus_{\mathrm{DP}}$ were analyzed using HP-SEC-RID-MALLS (Schatz et al. 2003), and DA was determined using hydrogen nuclear magnetic resonance $\left({ }^{1} \mathrm{H}-\mathrm{NMR}\right)$ (Hirai et al. 1991).

SEC analysis of the total hydrolysate showed the presence of a mixture of low molecular weight chitosan polymers and chitosan oligosaccharides (paCOS). The respective fractions were separated via centrifugal ultrafiltration with a membrane cutoff of $10 \mathrm{kDa}\left(4,000 \times \mathrm{g}, 15 \mathrm{~min}, 4^{\circ} \mathrm{C}\right)$ and yields of each fraction were estimated after freeze drying. Chitosan polymer was characterized for DP, $\mathrm{D}_{\mathrm{DP}}$, and $\mathrm{DA}$ as given above, and paCOS were analyzed using MALDI-TOF-MS (Schatz et al. 2003). 


\section{Preparation of $\boldsymbol{F}$. graminearum conidia.}

For the fungal pathogen, a strain of $F$. graminearum isolated in Wuhan (China) was used (Brunel et al. 2013) and conidia suspensions were prepared according to the method described by Hellweg (2003). F. graminearum was grown on complete medium (CM) agar plates for 10 days, transferred to a $200-\mathrm{ml}$ Erlenmeyer flask containing $50 \mathrm{ml}$ of CM, and incubated for 3 days at $26^{\circ} \mathrm{C}$ under continuous shaking at $120 \mathrm{rpm}$. Conidial production was induced by carboxymethyl cellulose medium. Conidia were harvested after 8 days of incubation at $26^{\circ} \mathrm{C}$ at $120 \mathrm{rpm}$ by filtration through cotton using an autoclaved glass funnel. The suspension was then centrifuged and washed with sterile water and the number of conidia per milliliter was assessed using a hemacytometer.

\section{Effect of chitosan on mycelial growth of $F$. graminearum.}

Antifungal activities of the parent chitosan, its hydrolysate, and the separated polymeric and oligomeric fractions of the hydrolysate were tested against $F$. graminearum in 96-well microtiter plates according to Oliveira et al. (2008). For these dose-response experiments, a volume of $40 \mu \mathrm{l}$ of chitosan solution at different concentrations or sterile water were added to $150 \mu \mathrm{l}$ of $\mathrm{CM}, \mathrm{pH}$

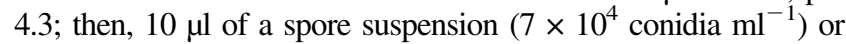
sterile water for the blanks was added to the mixture. Plates were incubated at $26^{\circ} \mathrm{C}$ under agitation for 5 days and fungal growth was assessed by measuring the optical density of the culture media at $600 \mathrm{~nm}$ using a UV/Vis-spectrophotometer (Multiscan GO 60; Thermo Scientific, Waltham, MA, U.S.A.) daily. Three replicates for each treatment were performed. The experiment was carried out three times independently.

The $\mathrm{IC}_{50}$ was defined as the concentration of each chitosan needed for inhibiting $50 \%$ of fungal growth. It was determined by nonlinear regression of the log-transformed values of inhibitor concentration $(\log [\mathrm{I}])$ versus the optical density at $600 \mathrm{~nm}$ using GraphPad Prism) (ver. 6; GraphPad Software, La Jolla, CA, U.S.A.).

The MIC was defined as the lowest concentration that fully inhibits fungal growth. It was determined visually as the lowest concentrations of the inhibitor at which no fungal growth was visible.

\section{Synergism between chitosan polymer and paCOS in inhibiting $F$. graminearum.}

To investigate the synergistic effect between chitosan polymer and paCOS, we combined an inactive concentration of paCOS $\left(50 \mu \mathrm{g} \mathrm{ml}^{-1}\right)$ with the following concentrations of the polymer: $0,10,30,50,70,90$, and $110 \mu \mathrm{g} \mathrm{ml}^{-1}$. paCOS and the polymer were also tested separately against $F$. graminearum as described in the section above. The interaction between the polymer and paCOS was quantified using two methods for synergy

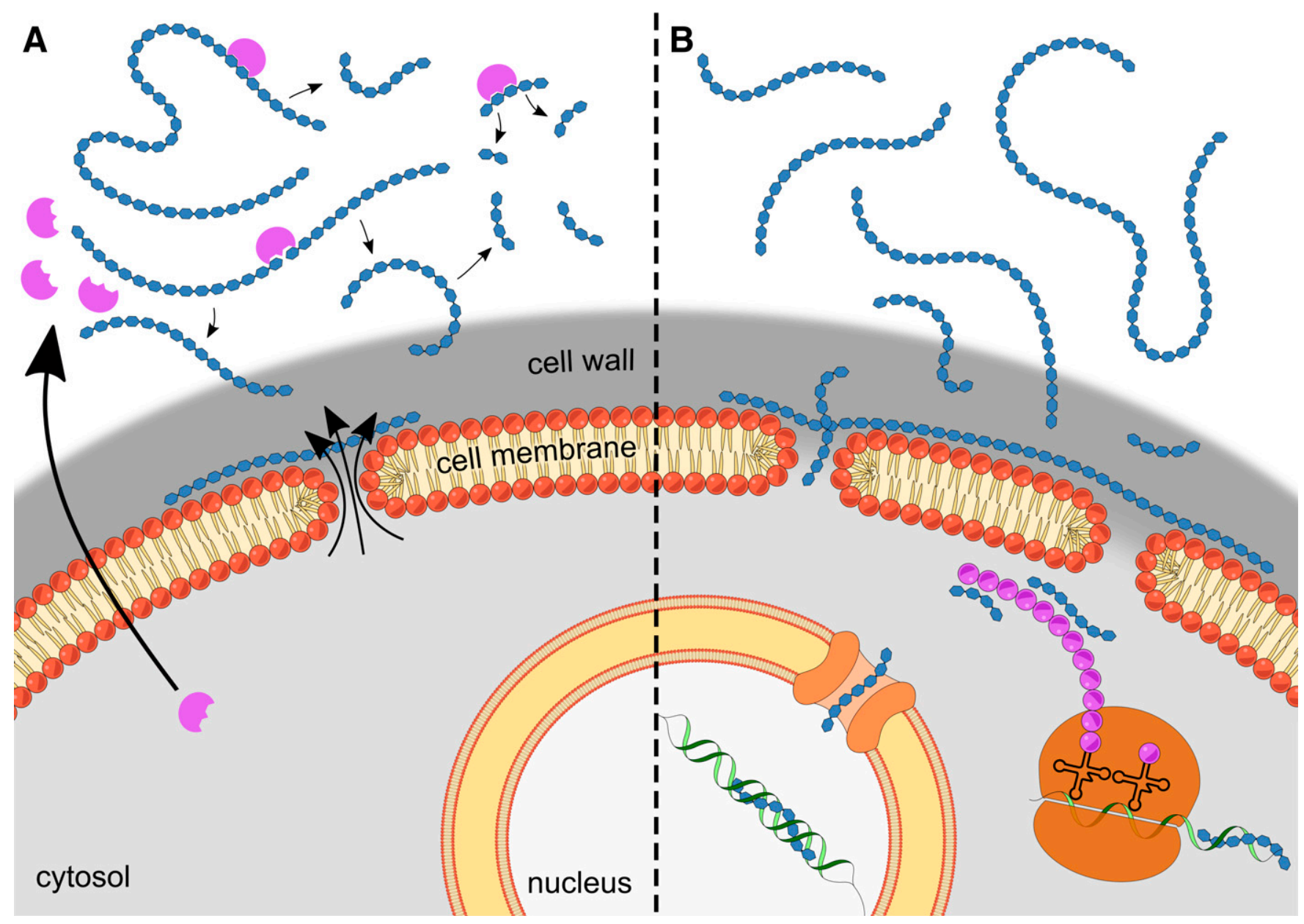

Fig. 6. Possible scenarios to explain the synergistic antifungal effect of chitosan polymers and oligomers (blue chains). A, Antifungal activity may arise from the large oligomers present in the mixture, which can penetrate the fungal cell wall and interact with the plasma membrane, destabilizing it and leading to the loss of intracellular components and, thus, weakening of the fungal pathogen. Chitosanolytic enzymes (purple) secreted by the fungus may degrade these antifungal oligomers, inactivating them, while the chitosan polymer may act like a reservoir from which the chitosan hydrolases slowly deliver a steady supply of antifungally active, large oligomers. B. Alternatively, the polymers may be able to penetrate the cell wall and interact with the membrane, destabilizing it and allowing chitosan oligomers to enter the fungal cell where they may interact with DNA, RNA, or proteins, perturbing their functions. 
calculation, the Abbott method for independent joint action and the Wadley method for similar joint action (Levy et al. 1986). The synergistic effect was calculated by determining the ratio between the observed efficacy (Eobs) (percent inhibition) and the expected efficacy (Eexp). An Eobs/Eexp ratio $=1$ indicates additivity, ratios $>1$ indicate synergy, and ratios $<1$ indicate antagonism.

\section{Time-lapse imaging.}

Aliquots containing CM, paCOS or polymer at $50 \mu \mathrm{g} \mathrm{ml}^{-1}$, or the mixture of paCOS and polymer at $50 \mu \mathrm{g} \mathrm{ml}^{-1}$ each, and fresh conidia were prepared as described above. Each treatment (30 $\mu \mathrm{l})$ was placed in a $\mu$-dish (Ibidi $\mathrm{GmbH}$, Munich, Germany), and images were taken with an inverted microscope (Axiovert Observer A1; Zeiss, Jena, Germany). We used the $\times 10$ objective lens and the bright-field channel to monitor the germination rates, and images were captured at 30-min intervals for 24 $\mathrm{h}$ with a Zeiss AxioCamMRm camera controlled by AxiovisionRel 4.8 software.

\section{Effect of chitosans on the morphology of $F$. graminearum.}

For SEM, hyphae were collected after 10 days of chitosan treatment and fixed in $2 \%$ glutaraldehyde overnight at $4^{\circ} \mathrm{C}$, rinsed three times with phosphate buffer $(0.1 \mathrm{M}, \mathrm{pH} 7)$, and subsequently fixed with $1 \%$ osmium tetroxide for $2 \mathrm{~h}$ at room temperature. The hyphae were dehydrated in a graded series of ethanol $\left(30,50,70,80,90\right.$, and 100\%) for 10 min each, $\mathrm{CO}_{2}$ dried, and sputter coated with gold palladium in a Nanotech sputter coating apparatus Emitec K550x (Emitec, Ashford, England). Samples were kept in a desiccator until examination with a Hitachi S-3 000N SEM (Hitachi Ltd. Co., Tokyo, Japan) operated at $25 \mathrm{kV}$.

\section{Chitosan hydrolase activity secreted by $F$. graminearum.}

A dot activity assay was conducted to investigate the presence of chitosan hydrolase in the spent media in the absence and presence of chitosans. F. graminearum was incubated at $26^{\circ} \mathrm{C}$ for 8 days in $\mathrm{CM}$ in the presence of paCOS or polymer at $50 \mu \mathrm{g}$ $\mathrm{ml}^{-1}$, or the mixture of paCOS and polymer at $50 \mu \mathrm{g} \mathrm{ml}$ each; medium without chitosan was used as a control. After 8 days, spent medium was collected by centrifugation and the supernatant was filtered using a 3-kDa filter. Protein concentration in the filtrates was determined using Bradford assay, and enzyme activity was tested in a dot activity assay (Nampally et al. 2012).

\section{ACKNOWLEDGMENTS}

We thank A. Norra for her help in the lab, K. Cohrs for his help with the time-lapse microscopy, S. Sreekumar for her help with the SEM, M. Bonin for his help with the illustration of the mode of action, and C. R. Brennecka from the Science Writing Support Service of the University of Münster for her editorial support.

\section{LITERATURE CITED}

Abbott, W. S. 1925. A method of computing the effectiveness of an insecticide. J. Econ. Entomol. 18:265-267.

Alburquenque, C., Bucarey, S. A., Neira-Carrillo, A., Urzúa, B., Hermosilla, G., and Tapia, C. V. 2010. Antifungal activity of low molecular weight chitosan against clinical isolates of Candida spp. Med. Mycol. 48:1018-1023.

Allan, C. R., and Hadwiger, L. A. 1979. The fungicidal effect of chitosan on fungi of varying cell wall composition. Exp. Mycol. 3:285-287.

Badawy, M. E., Ahmed, S. M., and Rabea, E. I. 2006. Bactericidal and fungicidal activities of different molecular weight chitosan samples. J. Pest Control Environ. Sci. 14:19-34.

Basa, S., Nampally, M., Honorato, T., Das, S. N., Podile, A. R., El Gueddari, N. E., and Moerschbacher, B. M. 2020. The pattern of acetylation defines the priming activity of chitosan tetramers. J. Am. Chem. Soc. 142:1975-1986.

Benhamou, N. 1992. Ultrastructural and cytochemical aspects of chitosan on Fusarium oxysporum f. sp. radicis-lycopersici, agent of tomato crown and root rot. Phytopathology 82:1185-1193.

Benhamou, N., and Theriault, G. 1992. Treatment with chitosan enhances resistance of tomato plants to the crown and root rot pathogen Fusarium oxysporum f. sp. radicis-lycopersici. Physiol. Mol. Plant Pathol. 41:33-52.

Brunel, F., El Gueddari, N. E., and Moerschbacher, B. M. 2013. Complexation of copper(II) with chitosan nanogels: Toward control of microbial growth. Carbohydr. Polym. 92:1348-1356.

Conrath, U., Domard, A., and Kauss, H. 1989. Chitosan-elicited synthesis of callose and of coumarin derivatives in parsley cell suspension cultures. Plant Cell Rep. 8:152-155.

Cord-Landwehr, S., Niehues, A., Wattjes, J., and Moerschbacher, B. M. 2020a. New developments in the analysis of partially acetylated chitosan oligomers and polymers. Pages 81-96 in: Chitin and Chitosan: Properties and Applications. L. A. M. van den Broek and C. G. Boeriu, eds. John Wiley \& Sons Ltd., Chichester, U.K.

Cord-Landwehr, S., Richter, C., Wattjes, J., Sreekumar, S., Singh, R., Basa, S., El Gueddari, N. E., and Moerschbacher, B. M. 2020 b. Patterns matter part 2: Chitosan oligomers with defined patterns of acetylation. React. Funct. Polym. 151:104577.

El Ghaouth, A., Arul, J., Grenier, J., and Asselin, A. 1992. Effect of chitosan and other polyions on chitin deacetylase in Rhizopus stolonifera. Exp. Mycol. 16:173-177.

El Gueddari, N. E., and Moerschbacher, B. M. 2004. A bioactivity matrix for chitosans as elicitors of disease resistance reactions in wheat. Adv. Chitin Sci. 7:56-59.

El Hadrami, A., Adam, L. R., El Hadrami, I., and Daayf, F. 2010. Chitosan in plant protection. Mar. Drugs 8:968-987.

Hadwiger, L. A., and Beckman, J. M. 1980. Chitosan as a component of pea-Fusarium solani interactions. Plant Physiol. 66:205-211.

Halim, A. S., Keong, L. C., Zainol, I., and Rashid, A. H. A. 2012. Biocompatibility and biodegradation of chitosan and derivatives. Pages 57-73 in: Chitosan-Based Systems for Biopharmaceuticals: Delivery, Targeting and Polymer Therapeutics. B. Sarmento and J. das Neves, eds. John Wiley \& Sons, Ltd., Chichester, U.K.

Hamer, S. N., Moerschbacher, B. M., and Kolkenbrock, S. 2014. Enzymatic sequencing of partially acetylated chitosan oligomers. Carbohydr. Res. 392:16-20.

Hellweg, M. 2003. Molecular biological and biochemical studies of proteolytic enzymes of the cereal pathogen Fusarium graminearum. Doctoral thesis, University of Münster.

Hernández-Lauzardo, A. N., Bautista-Baños, S., Velázquez-Del Valle, M. G., Méndez-Montealvo, M. G., Sánchez-Rivera, M. M., and Bello-Pérez, L. A. 2008. Antifungal effects of chitosan with different molecular weights on in vitro development of Rhizopus stolonifer (Ehrenb.:Fr.) Vuill. Carbohydr. Polym. 73:541-547.

Hirai, A., Odani, H., and Nakajima, A. 1991. Determination of degree of deacetylation of chitosan by ${ }^{1} \mathrm{H}$ NMR spectroscopy. Polym. Bull. 26:87-94.

Hirano, A., and Nagao, N. 1989. Effects of chitosan, pectic acid, lysozyme, and chitinase on the growth of several phytopathogens. Agric. Biol. Chem. 11:3065-3066.

Iriti, M., and Varoni, E. M. 2015. Chitosan-induced antiviral activity and innate immunity in plants. Environ. Sci. Pollut. Res. Int. 22:2935-2944.

Jeon, Y. J., Park, P. J., and Kim, S. K. 2001. Antimicrobial effect of chitooligosaccharides produced by bioreactor. Carbohydr. Polym. 44:71-76.

Kauss, H., Jeblick, W., and Domard, A. 1989. The degrees of polymerization and $\mathrm{N}$-acetylation of chitosan determine its ability to elicit callose formation in suspension cells and protoplasts of Catharanthus roseus. Planta 178:385-392.

Kheiri, A., Moosawi Jorf, S. A., Malihipour, A., Saremi, H., and Nikkhah, M. 2016. Application of chitosan and chitosan nanoparticles for the control of Fusarium head blight of wheat (Fusarium graminearum) in vitro and greenhouse. Int. J. Biol. Macromol. 93:1261-1272.

Kim, S.-K., and Rajapakse, N. 2005. Enzymatic production and biological activities of chitosan oligosaccharides (COS): A review. Carbohydr. Polym. 62:357-368.

Köhle, H., Jeblick, W., Poten, F., Blaschek, W., and Kauss, H. 1985. Chitosan-elicited callose synthesis in soybean cells as a ca-dependent process. Plant Physiol. 77:544-551.

Laflamme, P., Benhamou, N., Bussires, G., and Dessureault, M. 1999. Differential effect of chitosan on root rot fungal pathogens in forest nurseries. Can. J. Bot. 77:1460-1468. 
Levy, Y., Benderly, M., Cohen, Y., Gisi, U., and Bassand, D. 1986. The joint action of fungicides in mixtures: Comparison of two methods for synergy calculation. Bull. OEPP/EPPO Bull. 16:651-657.

Li, M., Chen, X., Liu, J., and Tang, X. 2011. Molecular weightdependent antifungal activity and action mode of chitosan against Fulvia fulva (Cooke) Ciffrri. J. Appl. Polym. Sci. 119:3127-3135.

Li, X. F., Feng, X. Q., Yang, S., and Wang, T. P. 2008. Effects of molecular weight and concentration of chitosan on antifungal activity against Aspergillus niger. Iran. Polym. J. 17:843-852.

Moerschbacher, B. M. 2005. Bio-activity matrices of chitosans in plant protection. Pages 186-190 in: Emerging Trends in Plant-Microbe Interactions. S. S. Gnanamanickam, R. Balasubramanian, and N. Anand, eds. University of Madras, Chennai, India.

Muñoz, I., Rodríguez, C., Gillet, D., and Moerschbacher, B. M. 2018. Life cycle assessment of chitosan production in India and Europe. Int. J. Life Cycle Assess. 23:1151-1160.

Nampally, M., Moerschbacher, B. M., and Kolkenbrock, S. 2012. Fusion of a novel genetically engineered chitosan affinity protein and green fluorescent protein for specific detection of chitosan in vitro and in situ. Appl. Environ. Microbiol. 78:3114-3119.

Naqvi, S., and Moerschbacher, B. M. 2017. The cell factory approach toward biotechnological production of high-value chitosan oligomers and their derivatives: An update. Crit. Rev. Biotechnol. 37:11-25.

Nars, A., Rey, T., Lafitte, C., Vergnes, S., Amatya, S., Jacquet, C., Dumas, B., Thibaudeau, C., Heux, L., Bottin, A., and Fliegmann, J. 2013. An experimental system to study responses of Medicago truncatula roots to chitin oligomers of high degree of polymerization and other microbial elicitors. Plant Cell Rep. 32:489-502.

Niekraszewicz, A., Wiśniewska-Wrona, M., Kopania, E., Orlikowski, L., Pospieszny, H., and Krawczyk, K. 2012. Biopolymer compositions for ecological protection and growth stimulation of plants. Prog. Chem. Appl. Chitin Deriv. 17:149-162.

Oliveira, E. N., Jr., El Gueddari, N. E., Moerschbacher, B. M., Peter, M. G., and Franco, T. T. 2008. Growth of phytopathogenic fungi in the presence of partially acetylated chitooligosaccharides. Mycopathologia 166:163-174.

Orzali, L., Forni, C., and Riccioni, L. 2014. Effect of chitosan seed treatment as elicitor of resistance to Fusarium graminearum in wheat. Seed Sci. Technol. 42:132-149.

Palma-Guerrero, J., Huang, I. C., Jansson, H. B., Salinas, J., LopezLlorca, L. V., and Read, N. D. 2009. Chitosan permeabilizes the plasma membrane and kills cells of Neurospora crassa in an energy dependent manner. Fungal Genet. Biol. 46:585-594.

Park, Y., Kim, M. H., Park, S. C., Cheong, H., Jang, M. K., Nah, J. W., and Hahm, K. S. 2008. Investigation of the antifungal activity and mechanism of action of LMWS-chitosan. J. Microbiol. Biotechnol. 18:1729-1734.

Plascencia-Jatomea, M., Viniegra, G., Olayo, R., Castillo-Ortega, M. M., and Shirai, K. 2003. Effect of chitosan and temperature on spore germination of Aspergillus niger. Macromol. Biosci. 3:582-586.

Putriningrum, R., Nurhidayati, A., Umarianti, T., Listyaningsih, K. D., and Agustin, W. R. 2016. The synergistic effects of Areca nut extract and chitosan toward Candida albicans in vitro. Int. J. Pharma Med. Biol. Sci. 2:142-145.

Raafat, D., and Sahl, H. G. 2009. Chitosan and its antimicrobial potential-a critical literature survey. Microb. Biotechnol. 2:186-201.

Rabea, E. I., Badawy, M. E. T., Stevens, C. V., Smagghe, G., and Steurbaut, W. 2003. Chitosan as antimicrobial agent: Applications and mode of action. Biomacromolecules 4:1457-1465.

Rahman, M. H., Shovan, L. R., Hjeljord, L. G., Aam, B. B., Eijsink, V. G. H., Sørlie, M., and Tronsmo, A. 2014. Inhibition of fungal plant pathogens by synergistic action of chito-oligosaccharides and commercially available fungicides. PLoS One 9:e93192.

Reddy, M. V. B., Arul, J., Ait-Barka, E., Angers, P., Richard, C., and Castaigne, F. 1998. Effect of chitosan on growth and toxin production by Alternaria alternata f. sp. lycopersici. Biocontrol Sci. Technol. 8:33-43.

Reddy, M. V. B., Arul, J., Angers, P., and Couture, L. 1999. Chitosan treatment of wheat seeds induces resistance to Fusarium graminearum and improves seed quality. J. Agric. Food Chem. 47:1208-1216.

Rhoades, J., and Roller, S. 2000. Antimicrobial actions of degraded and native chitosan against spoilage organisms in laboratory media and foods. Appl. Environ. Microbiol. 66:80-86.

Roller, S., and Covill, N. 1999. The antifungal properties of chitosan in laboratory media and apple juice. Int. J. Food Microbiol. 47:67-77.

Schatz, C., Viton, C., Delair, T., Pichot, C., and Domard, A. 2003. Typical physicochemical behaviors of chitosan in aqueous solution. Biomacromolecules 4:641-648.

Shigemasa, Y., and Minami, S. 1996. Applications of chitin and chitosan for biomaterials. Biotechnol. Genet. Eng. Rev. 13:383-420.

Sudarshan, N. R., Hoover, D. G., and Knorr, D. 1992. Antibacterial action of chitosan. Food Biotechnol. 6:257-272.

Tsai, G. J., and Su, W. H. 1999. Antibacterial activity of shrimp chitosan against Escherichia coli. J. Food Prot. 62:239-243.

van den Broek, L. A. M., and Boeriu, C. G. 2020. Chitin and Chitosan: Properties and Applications. John Wiley \& Sons Ltd., Chichester, U.K.

Vander, P., Vårum, K. M., Domard, A., El Gueddari, N. E., and Moerschbacher, B. M. 1998. Comparison of the ability of partially $\mathrm{N}$-acetylated chitosans and chitooligosaccharides to elicit resistance reactions in wheat leaves. Plant Physiol. 118:1353-1359.

Wadley, F. M. 1945. The evidence required to show synergistic action of insecticides and a short cut in analysis. ET Circ. 223. U.S. Department of Agriculture, U.S. Government Printing Office, Washington, DC, U.S.A.

Wattjes, J., Sreekumar, S., Richter, C., Cord-Landwehr, S., Singh, R., El Gueddari, N. E., and Moerschbacher, B. M. 2020. Patterns matter part 1: Chitosan polymers with non-random patterns of acetylation. React. Funct. Polym. 151:104583.

Winkler, A. J., Dominguez-Nuñez, J. A., Aranaz, I., Poza-Carrión, C., Ramonell, K., Somerville, S., and Berrocal-Lobo, M. 2017. Shortchain chitin oligomers: Promoters of plant growth. Mar. Drugs 15:40.

Younes, I., and Rinaudo, M. 2015. Chitin and chitosan preparation from marine sources. Structure, properties and applications. Mar. Drugs 13:1133-1174. 